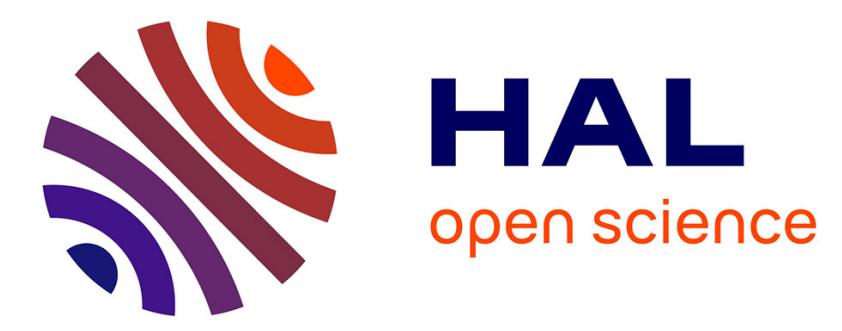

\title{
Application de l'analyse Auger à l'étude des surfaces de films obtenus en milieu aqueux. Application aux dépôts de nickel chimique
}

\author{
S. Goldsztaub, B. Carrière, J. Flechon, F. Machizaud
}

\section{- To cite this version:}

S. Goldsztaub, B. Carrière, J. Flechon, F. Machizaud. Application de l'analyse Auger à l'étude des surfaces de films obtenus en milieu aqueux. Application aux dépôts de nickel chimique. Revue de Physique Appliquée, 1976, 11 (1), pp.51-55. 10.1051/rphysap:0197600110105100 . jpa-00244042

\section{HAL Id: jpa-00244042 https://hal.science/jpa-00244042}

Submitted on 1 Jan 1976

HAL is a multi-disciplinary open access archive for the deposit and dissemination of scientific research documents, whether they are published or not. The documents may come from teaching and research institutions in France or abroad, or from public or private research centers.
L'archive ouverte pluridisciplinaire HAL, est destinée au dépôt et à la diffusion de documents scientifiques de niveau recherche, publiés ou non, émanant des établissements d'enseignement et de recherche français ou étrangers, des laboratoires publics ou privés. 


\title{
APPLICATION DE L'ANALYSE AUGER A L'ÉTUDE DES SURFACES DE FILMS OBTENUS EN MILIEU AQUEUX APPLICATION AUX DÉPÔTS DE NICKEL CHIMIQUE
}

\author{
S. GOLDSZTAUB, B. CARRIÈRE
}

Laboratoire de Minéralogie, U. L. P. Strasbourg E. R. A. № 07, France

J. FLECHON et F. MACHIZAUD

Laboratoire de Physique des Dépôts Métalliques, Univ. Nancy I, France

\begin{abstract}
Résumé. - L'étude des films préparés par oxydoréduction en milieu aqueux est possible par spectrométrie Auger.

Les spectres obtenus sont caractéristiques des dépôts et de leur contamination.

Nous indiquons les premières corrélations entre ces spectres et les propriétés électriques, magnétiques et structurales des dépôts.
\end{abstract}

\begin{abstract}
The study of films $\mathrm{Ni}, \mathrm{P}$ deposited by oxydo reduction from aqueous solutions si possible by spectrometry Auger.

The diagrams are characteristic of the alloy and his contamination.

We give the first correlations with the results of the electrical, magnetic and structural investigations.
\end{abstract}

1. Introduction. - L'analyse des surfaces par spectrométrie Auger porte habituellement sur des monocristaux, des couches minces obtenues par vaporisation sous vide ou des solides polycristallins.

Dans tous les cas on évite la contamination des surfaces due soit à l'enceinte, soit à la préparation ellemême, par un étuvage à $250^{\circ} \mathrm{C}$ suivi d'un clivage ou fracture dans l'ultra-vide. On peut également procéder à un bombardement ionique. On peut enfin étudier la contamination sans traitement spécial.

L'ensemble des précautions nécessaires à l'obtention d'une surface propre semblait écarter systématiquement toute étude par spectrométrie Auger de matériaux obtenus à l'air libre, en phase liquide aqueuse et dont la structure, évoluant avec la température, ne permettait pas l'étuvage préalable à tout examen.

Il était donc tentant de tester des films d'alliages obtenus par oxydo-réduction dans des solutions dont la température n'excède jamais $90^{\circ} \mathrm{C}$. C'est en particulier le cas des dépôts de Ni-P.

2. Préparation des films. - De nombreuses études $[1,2,3,4,5]$ tant chimiques que physiques ont été conduites sur ces substances dont l'état cristallin très imparfait est à l'origine de propriétés électriques [6] et magnétiques [7] très particulières.

La méthode de préparation est bien connue :

Une solution aqueuse d'un sel de $\mathrm{Ni}$ est réduite par l'hypophosphite de $\mathrm{Na}$ en présence de traces de chlo- rure de Pd. Une certaine masse de métal et de métalloïde est libérée et se fixe sur tout support isolant immergé dans le liquide, en particulier des lames de silice ou de verre. Le film obtenu possède l'éclat métallique et une rigidité suffisante pour être éventuellement détaché de son support, son épaisseur varie de quelques centaines à quelques milliers d'angströms. Il est formé de $\mathrm{Ni}$ et de 3 à $12 \%$ de $\mathrm{P}$ en masse, suivant les conditions expérimentales.

3. Caractères physiques. - En l'absence de tout traitement thermique un tel matériau est essentiellement constitué par une phase solide amorphe où $\mathrm{Ni}$ et $P$ sont liés sans qu'une espèce chimique, telle qu'un phosphure de $\mathrm{Ni}$, puisse être clairement isolée, et des traces de $\mathrm{Ni}$. L'existence de liaisons entre ces deux éléments a été mise en évidence par une déphosphoration chimique [3].

La microdiffraction électronique comme le spectre $X$ (Fig. 1) confirment ces faits : des halos de diffraction indiquent la présence d'une phase amorphe.

De même, la conductivité électrique $\sigma$ est environ 100 fois plus faible que celle d'une lame de Ni de même épaisseur, soit $1,3 \times 10^{7} \mathrm{~s} . \mathrm{m}^{-1}$. Le coefficient de température moyen des résistances $K_{\theta}$ est inférieur à $200 \times 10^{-6}$, soit 20 fois moins que celui de $\mathrm{Ni}$ pur. L'aimantation à saturation $J_{\mathrm{s}}$ de la matrice dans un champ de 2,5 $\mathrm{T}$ est, pour la composition étudiée, $0,9 \times 10^{5} \mathrm{~A} / \mathrm{m}\left(J_{\mathrm{s}} \mathrm{du} \mathrm{Ni}\right.$ pur est $\left.4,2 \times 10^{5} \mathrm{~A} / \mathrm{m}\right)$. 


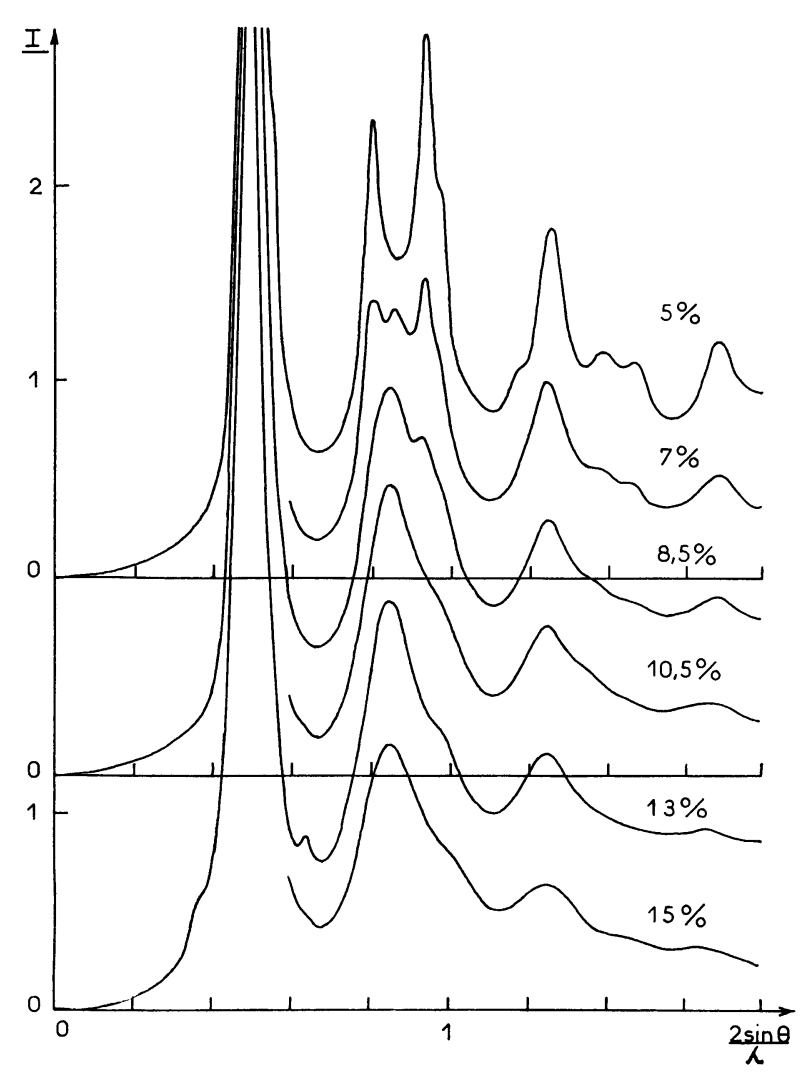

Fig. 1. - Diagramme de diffraction $\mathrm{X}$ de l'alliage Ni-P (5\%$15 \% \mathrm{P})$ à $50^{\circ} \mathrm{C}$.

Dès que la température s'élève de l'ambiante à $500^{\circ}$ (Fig. 2) la structure s'organise ; la résistivité décroît et peut atteindre le $30^{\mathrm{e}}$ de sa valeur initiale. Le coefficient

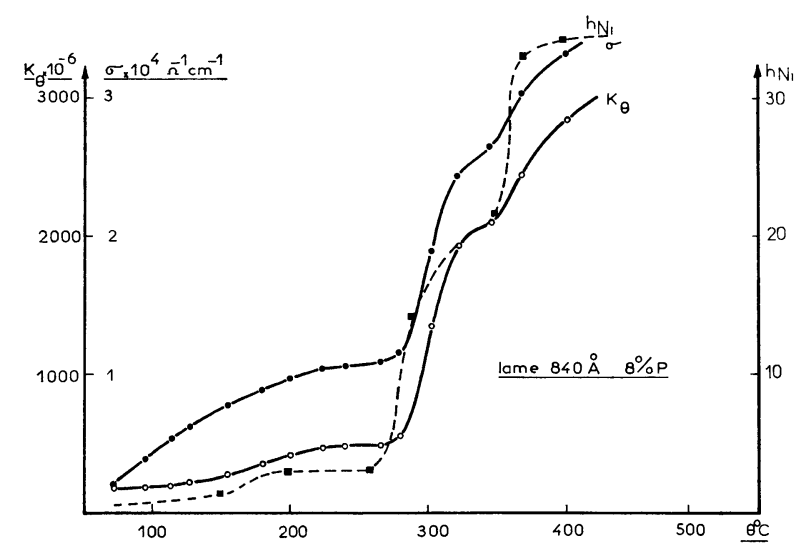

Fig. 2. - Influence de la température sur la conductivité $\sigma$, le coefficient de température $K_{\theta}$ et la hauteur $h_{\mathrm{Ni}}$ du pic Auger du Ni de l'alliage Ni-P à $8 \% \mathrm{P}$.

de température augmente jusqu'à 30 fois $K_{\theta}$ initial, l'aimantation à saturation $J_{\mathrm{s}}$ est triplée (Fig. 3).

L'examen des spectres $\mathrm{X}$ permet d'interpréter l'évolution de la matrice : une séparation progressive des deux phases $\mathrm{Ni}$ et $\mathrm{Ni}_{3} \mathrm{P}$. En effet, à $8,5 \%$ en masse, correspondent dans $100 \mathrm{~g}$ de substance, $48 \mathrm{~g}$ de $\mathrm{Ni}$

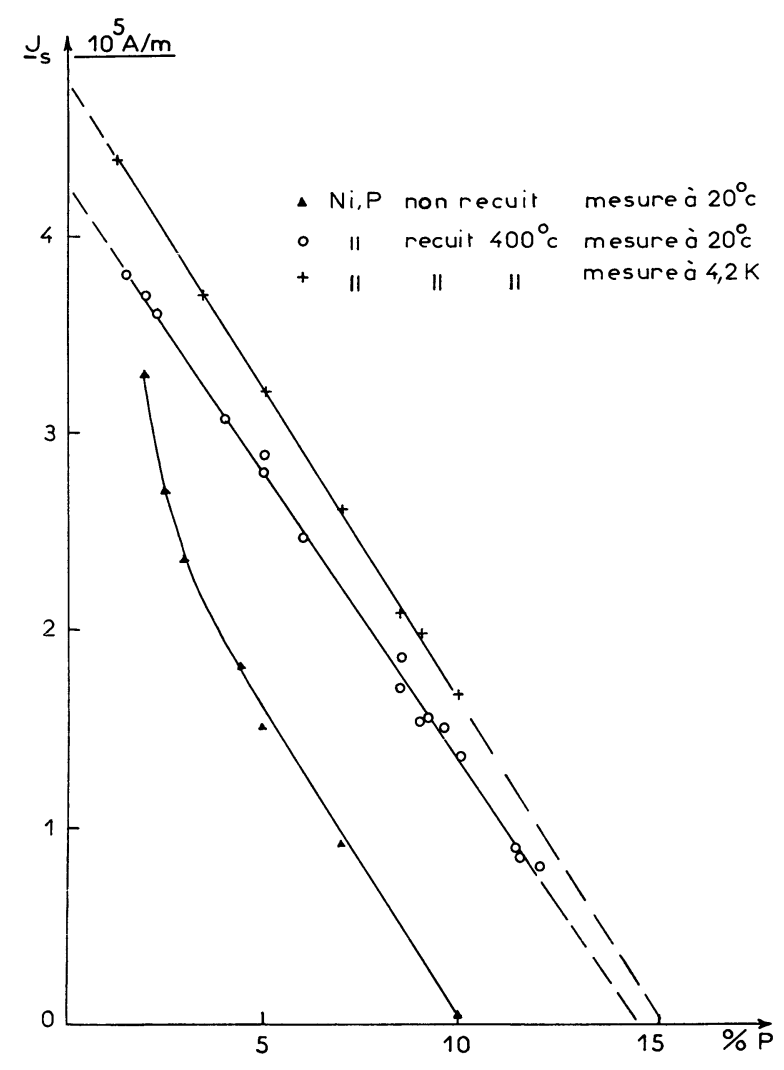

Fig. 3. - Influence de la teneur en $\mathrm{P}$ sur l'aimantation à saturation $J_{\mathrm{S}}$ de l'alliage Ni-P [7].

combinés au métalloïde en admettant que tout le phosphore s'unisse au métal. Il reste donc $43,5 \mathrm{~g}$ de Ni libre.

Ainsi le processus est le suivant :

Dès $150^{\circ} \mathrm{C}$ séparation de microcristaux de Ni détectables par les raies $\mathrm{X}$ caractéristiques.

Au-delà de $300^{\circ} \mathrm{C}$ (Fig. 4) précipitation de $\mathrm{Ni}_{3} \mathrm{P}$ révélée par son spectre $X$. A partir de $350^{\circ}$ augmentation de la taille des cristaux des deux phases.

On constate donc que les propriétés physiques étudiées évoluent lorsque la température s'élève.

Les résultats précédents concernent les propriétés volumiques. Nous avons tenté de les compléter par l'étude de la surface à l'aide de l'analyse Auger en faisant subir à l'échantillon les mêmes traitements thermiques que précédemment.

4. Analyse par électrons Auger. - 4.1 AppareilLAGE [ET MODE OPÉRATOIRE. - L'analyseur utilisé fonctionne sur le principe du champ retardateur et utilise l'optique à 4 grilles d'un diffractographe à électrons lents. La figure 5 montre le schéma de cet analyseur. On enregistre les courbes $\mathrm{d} N(E) / \mathrm{d} E$ qui permettent une meilleure détermination de l'énergie des pics Auger et des déplacements qu'ils peuvent subir. On mesure par convention l'énergie du pic en son point d'ordonnée minimale sur la courbe $\mathrm{d} N(E) / \mathrm{d} E$ et sa grandeur $h$ par la différence des ordonnées des deux extrema. 


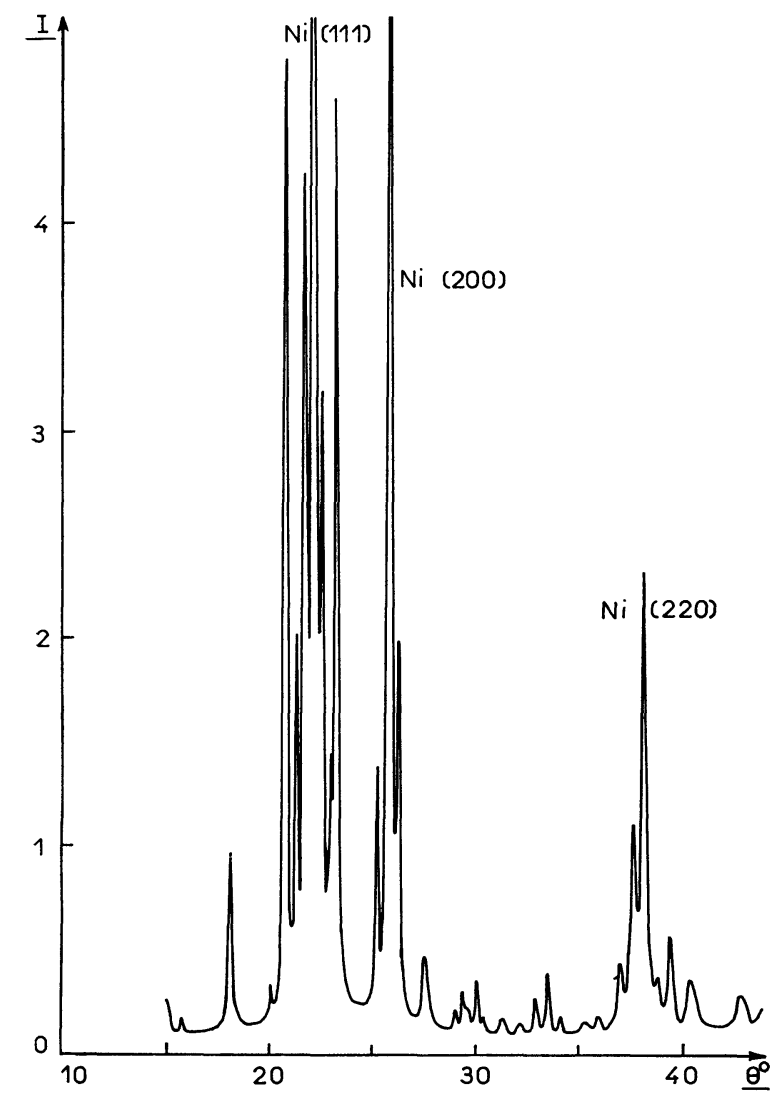

FIG 4. - Diagramme de diffraction $\mathrm{X}$ (rayonnement $\mathrm{Cu}_{\mathrm{k} \alpha}$ ) de l'alliage Ni-P $8,5 \% \mathrm{P}$ à $450^{\circ} \mathrm{C}$.

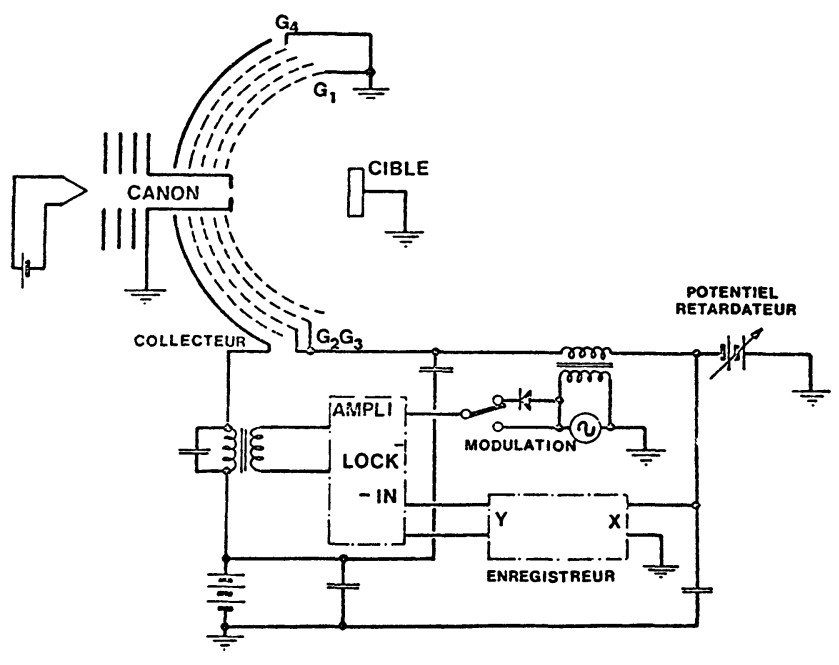

Fig. 5. - Schéma de l'analyseur Auger.

Nous avons considéré l'évolution, en fonction de la température :

1) de l'énergie des pics de $\mathrm{Ni}$ et de $\mathrm{P}$,

2) des rapports $h_{\mathrm{Ni}} / h_{\mathrm{C}}$ et $h_{\mathrm{P}} / h_{\mathrm{C}}$.

L'énergie primaire $E_{\mathrm{p}}$ constante tout au long de l'étude est de $1000 \mathrm{eV}$, le débit $I_{\mathrm{p}}$ est compris entre 4 et $9 \times 10^{-6}$ A. Le choix d'une modulation constante $(1,5 \mathrm{~V}$ crête à crête pour le $\mathrm{Ni}, 3 \mathrm{~V}$ crête à crête pour le $\mathrm{P}$ et le $\mathrm{C}$ ) est nécessaire pour pouvoir raisonner sur la variation des hauteurs respectives des pics de $\mathrm{Ni}, \mathrm{P}$ et $\mathrm{C}$ et de leurs rapports. Les hauteurs mesurées sont rapportées à un débit constant.

Les échantillons étudiés ont une épaisseur de l'ordre de $900 \AA ̊$ et contiennent environ $8 \%$ du P ; ils ont été simplement lavés et séchés dans un excicateur avant d'être introduits dans l'enceinte. Ils y sont fixés avec leur support de verre entre deux rubans de Ta qui permettent leur chauffage par effet Joule.

Un étuvage de douze heures à $150^{\circ} \mathrm{C}$ permet d'obtenir un vide de $10^{-9}$ torr. L'ensemble des mesures a été effectué aux températures de traitement.

4.2 RÉSUltats. - Le premier spectre, après étuvage, est enregistré à $150^{\circ} \mathrm{C}$ et présente des pics peu intenses à 57,5 et $114,5 \mathrm{eV}$ dus au $\mathrm{Ni}$ et au $\mathrm{P}$, un pic de $\mathrm{C}$ très important à $270 \mathrm{eV}$. D'autres pics beaucoup plus faibles, difficilement interprétables, pourraient être dus notamment au $\mathrm{S}$ et au $\mathrm{Cl}$ (Fig. 6). On peut noter que les énergies des pics attribués au Ni et au P sont nettement déplacées par rapport à celles (61-62 eV pour le $\mathrm{Ni}$ et 119,5 eV pour le $P$ ) données dans la littérature pour ces éléments $[8,9]$.

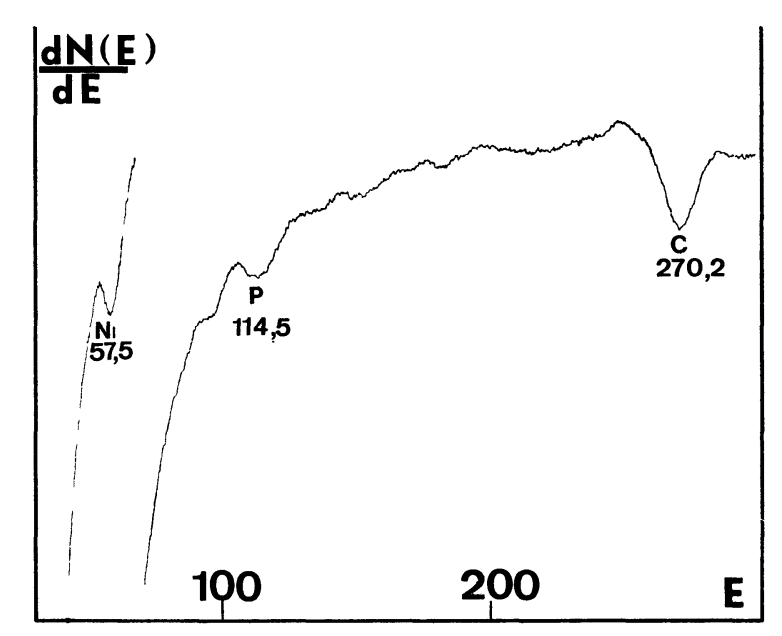

Fig. 6. - Spectre Auger de l'alliage Ni-P obtenu à $150^{\circ} \mathrm{C}$ après étuvage.

Nous avons procédé ensuite à des chauffages isothermes successifs analogues à ceux pratiqués lors des études physiques antérieures. La température de travail passe par palier de 150 à $450^{\circ} \mathrm{C}$. Les spectres Auger sont enregistrés pour une température $T$ après un temps de chauffage qui correspond à la stabilisation des propriétés électriques de ces lames.

La figure 7 montre l'évolution des énergies des pics de Ni et P, la figure 8, celle des rapports $h_{\mathrm{Ni}} / h_{\mathrm{C}}$ et $h_{\mathrm{P}} / h_{\mathrm{C}^{-}}$.

$\mathrm{Si}$ nous considérons ces résultats pour les températures supérieures à $300^{\circ} \mathrm{C}$, température à partir de laquelle la contamination diminue notablement, on constate (Fig. 7) que le lieu des extrema pour Ni comme pour $\mathbf{P}$ présente deux paliers séparés par une disconti- 


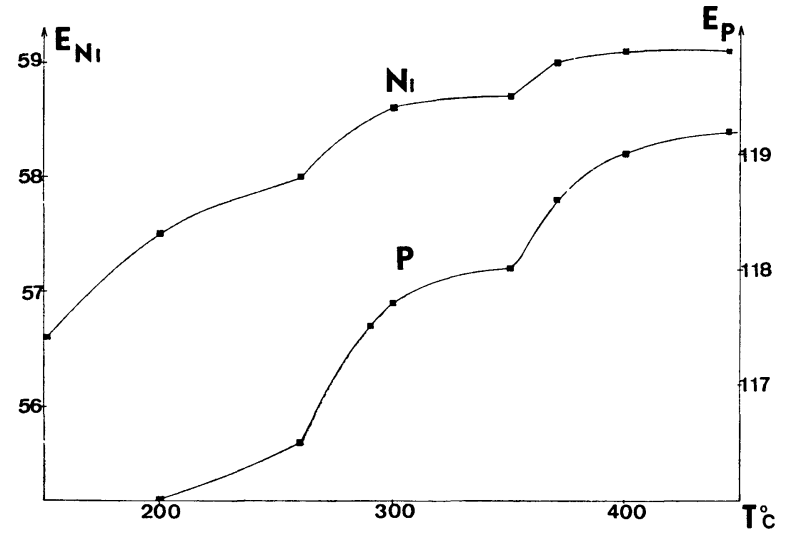

Fig. 7. - Evolution des énergies des pics Auger de $\mathrm{Ni}$ et $\mathrm{P}$ en fonction de la température.

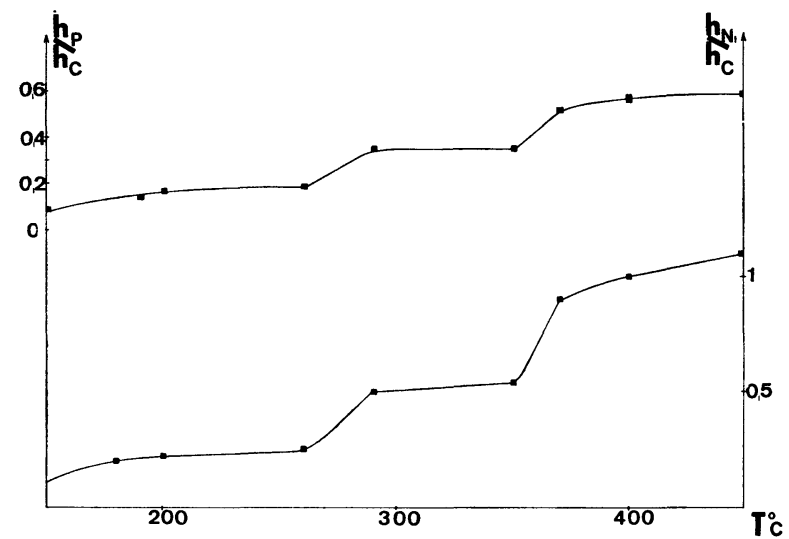

FIG. 8. - Evolution des rapports des hauteurs des pics $h_{\mathrm{N} i} / h_{\mathrm{C}}$ et $h_{\mathrm{P}} / h_{\mathrm{C}}$ en fonction de la température.

nuité, ce que nous pouvons résumer dans le tableau ci-dessous

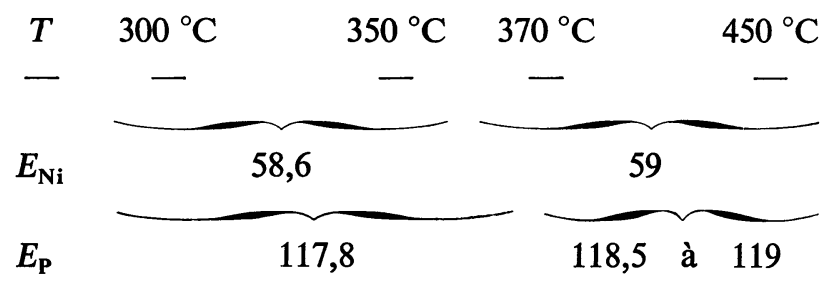

Ces résultats correspondent aux variations de conductivité et du coefficient de température.

Jusqu'à $300^{\circ} \mathrm{C}$ les pics caractéristiques de $\mathrm{Ni}$ et $\mathrm{P}$ se déplacent progressivement. Le premier palier $\left(300^{\circ}\right.$ à $350^{\circ} \mathrm{C}$ ) est associé à la précipitation de $\mathrm{Ni}$ et $\mathrm{Ni}_{3} \mathrm{P}$, le second à la croissance cristalline des mêmes phases.

Si nous considérons la figure 8 , on observe au-delà de $290^{\circ} \mathrm{C}$ deux paliers analogues à ceux des énergies et limites aux mêmes températures. Les corrélations précédemment soulignées se retrouvent, le dernier palier devant correspondre à un enrichissement superficiel en $\mathrm{Ni}$ et $\mathrm{P}$.

Enfin, si nous portons sur le même diagramme le coefficient de température, la conductivité et la hauteur du pic de Ni (Fig. 2), nous constatons une correspondance significative entre ces trois grandeurs.

Le spectre de la figure 9 a été enregistré pour une température de $350^{\circ} \mathrm{C}$. On constate par rapport au spectre de la figure 6 le déplacement des pics de Ni et $\mathrm{P}$, et l'augmentation de leurs grandeurs, sans que la diminution du pic de carbone soit encore très importante.

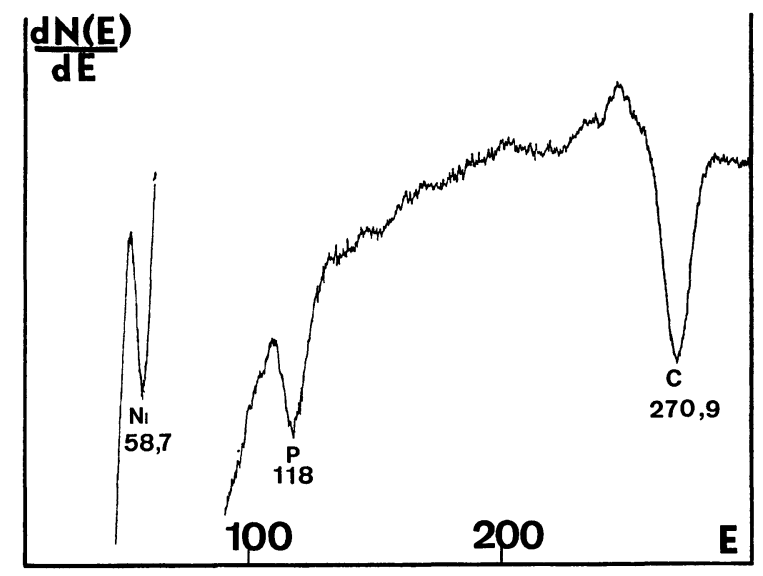

FIG. 9. - Spectre Auger de l'alliage Ni-P obtenu à $350^{\circ} \mathrm{C}$.

Le spectre finalement obtenu à $450^{\circ} \mathrm{C}$ (Fig. 10) est caractéristique d'une surface notablement décontaminée sur laquelle le $\mathrm{Ni}$ et le $\mathrm{P}$ sont dans un état de liaison plus proche de l'état élémentaire $\left(E_{\mathrm{Ni}}=61\right.$ et $\left.E_{\mathrm{P}}=119,5 \mathrm{eV}\right)$.

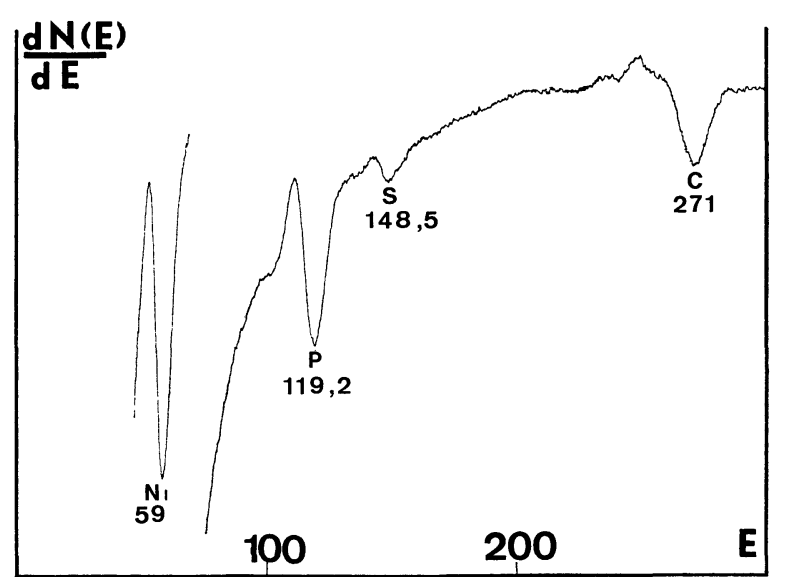

FIg. 10. - Spectre Auger de l'alliage Ni-P obtenu à $450^{\circ} \mathrm{C}$.

Il est intéressant de noter, outre les correspondances entre les résultats de l'analyse Auger et des études physiques antérieures, que des chauffages à des températures inférieures à $500^{\circ} \mathrm{C}$ ont permis un bon nettoyage de ces surfaces.

5. Conclusion. - Cette première étude montre qu'il est possible d'examiner par spectrométrie Auger des matériaux qui n'ont pas subi les traitements ordinaires 
que requiert cette technique. C'est le cas des films minces de nickel-phosphore.

L'évolution structurale de ces substances avec la température entraîne une modification des propriétés électriques et magnétiques en corrélation avec la position des pics Auger du nickel et du phosphore et avec leurs intensités. La position des pics de nickel et de phosphore présents dans la phase amorphe et leur déplacement avec la température soulignent l'existence et l'évolution des liaisons chimiques entre ces deux éléments sans que l'on puisse en préciser la nature.
Pour compléter cette étude, il faudrait :

1) Comparer les spectres du $\mathrm{Ni}$ pur et de $\mathrm{Ni}_{3} \mathrm{P}$ enregistrés dans les mêmes conditions, à ceux de ces films.

2) Etudier la variation des hauteurs des pics du nickel et du phosphore en fonction de la composition de l'échantillon.

3) Essayer de décaper la surface par bombardement ionique, pour comparer les compositions superficielles et volumiques de ces substances.

\section{Bibliographie}

[1] Brenner, A. et Riddel, G. E., J. Res. Nat. Bur. Stand. [5] Randin, J. P., Thèse (1969) Neufchâtel.

$$
\text { (1946) } 371
$$

[2] Gutzert, G., Plating 46 (1959) $1275 ; 47$ (1960) 63.

[3] FleChON, J., Thèse (1960) Nancy.

[4] DixMIER, J., Thèse (1969) Orsay.
[6] Machizaud, F., Thèse (1973) Nancy.

[7] Viard, M., Thèse (1974) Nancy.

[8] Demuth, J. E. et Rhodin, T. N., SS 42 (1974) 272.

[9] Morgan, A. E. et van Velzen, W. J. M., SS 40 (1973) 360. 